\title{
Towards a Unified Theory of Regulated Grammars
}

\author{
Sherzod Turaev \\ Research Group on Mathematical Linguistics \\ Universitat Rovira i Virgili \\ Tarragona, Spain \\ E-mail: sherzod.turaev@urv.cat
}

Summary. This paper shows that Petri net control mechanisms in grammars can be considered to be a unifying approach to different controls and characterizations which are used in well-known families of languages such as matrix and vector languages.

\section{Introduction}

It is well-known that context-free languages are not sufficient to model phenomena which are known in natural and programming languages. On the other hand, context-sensitive languages are much more powerful and have negative features (e.g. among decision problems only membership is decidable and all existing algorithms for this problem have exponential complexities). Therefore, the languages of interest are those that use context-free production rules and have larger generative capacities. Many grammars have a context-free base and a mechanism that controls the sequences of rules in a derivation $[1,3,4,5,8,10,14,15]$.

A context-free grammar and its derivation process can be described by a Petri net, where places correspond to nonterminals and terminals, transitions are the counterpart of productions, and tokens reflect the occurrences of symbols in the 
sentential form, and there is a one-to-one correspondence between the application of (sequences of) rules and the firing of (sequences of) transitions. Therefore, controlling the sequences of rules in a derivation by adding some features to the associated Petri net is natural and straightforward.

In [2] it has been shown that by adding some places and arcs which satisfy some structural requirements, random context languages can be generated. In this paper we show that vector, semi-matrix and matrix languages can also be generated by grammars controlled by Petri nets. Thus, control by Petri nets can be considered as a unifying approach to different types of controls and characterizations used in well-known language families.

The paper is organized as follows. In Section 2 we give some notions and definitions from the theories of formal languages and the Petri nets needed in the sequel. We also introduce the Petri net associated with a context-free grammar. In Section 3 we construct the Petri net control mechanisms, define the corresponding grammars, and give some examples. Section 4 contains the results on the generative powers of families of languages generated by Petri net controlled grammars.

\section{Definitions}

The reader is assumed to be familiar with the basic notions of formal language theory and Petri net theory contained in $[4,6,11,7,9,12,13]$.

\subsection{Grammars}

Let $\Sigma=\left\{a_{1}, a_{2}, \ldots, a_{k}\right\}$ be an alphabet. A string over $\Sigma$ is a sequence of symbols from the alphabet. If $w=w_{1} w_{2} w_{3}$ for some $w_{1}, w_{2}, w_{3} \in \Sigma^{*}$, then $w_{2}$ is called a substring of $w$. A word $u=u_{1} u_{2} \cdots u_{n}, u_{1}, u_{2}, \ldots, u_{n} \in \Sigma$ is a scattered substring of $v \in \Sigma^{*}$, denoted by $u \ll v$, if $v=v_{1} u_{1} v_{2} \cdots u_{n} v_{n+1}$ for some $v_{1}, v_{2}, \ldots, v_{n+1} \in \Sigma^{*}$ with $n \geq 1$. We write $u \nless k$ if $u$ is not a scattered substring of $v$. The length of a string $w$ is denoted by $|w|$, and the number of occurrences of a symbol $a$ in a string $w$ by $|w|_{a}$. The empty string is denoted by $\lambda$ which is of length 0 . The set of all strings over the alphabet $\Sigma$ is denoted by $\Sigma^{*}$. A subset $L$ of $\Sigma^{*}$ is called a language.

For $u, v \in \Sigma^{*}$ their shuffle is defined by $\operatorname{sh}(u, v)=u_{1} v_{1} u_{2} v_{2} \cdots u_{n} v_{n}$ where $u=u_{1} u_{2} \cdots u_{n}$ and $v=v_{1} v_{2} \cdots v_{n}, u_{i}, v_{i} \in \Sigma^{*}, 1 \leq i \leq n$. A shuffle of $u$ and $v$ is proper if neither $\operatorname{sh}(u, v)=u v$ nor $\operatorname{sh}(u, v)=v u$. A proper shuffle is denoted by $\operatorname{sh}^{+}(u, v)$. The operation shuffle can be generalized for more than two strings by $\operatorname{sh}\left(u_{1}, u_{2}, \ldots, u_{n}\right)=\operatorname{sh}\left(\operatorname{sh}\left(u_{1}, u_{2}, \ldots, u_{n-1}\right), u_{n}\right), n \geq 3$. A shuffle of $u_{1}, u_{2}, \ldots, u_{n}$, $n \geq 2$, is semi, denoted by $\operatorname{ssh}\left(u_{1}, u_{2}, \ldots, u_{n}\right)$, if $u_{i}=u_{j}$, for some $1 \leq i<j \leq$ $n$, then $\operatorname{sh}^{+}\left(u_{i}, u_{j}\right) \nless \operatorname{sh}\left(u_{1}, u_{2}, \ldots, u_{n}\right)$. In words, a semi-shuffle string does not contain self-shuffled scattered substrings. 
A context-free grammar is a quadruple $G=(V, \Sigma, S, R)$ where $V$ and $\Sigma$ are the disjoint finite sets of nonterminal and terminal symbols, respectively, $S \in V$ is the start symbol and the finite set $R \subset V \times(V \cup \Sigma)^{*}$ is the set of (production) rules. Usually, a rule $(A, x)$ is written as $A \rightarrow x$. A rule of the form $A \rightarrow \lambda$ is called an erasing rule. The word $x \in(V \cup \Sigma)^{+}$directly derives $y \in(V \cup \Sigma)^{*}$, written as $x \Rightarrow y$, iff there is a rule $r=A \rightarrow \alpha \in R$ such that $x=x_{1} A x_{2}$ and $y=x_{1} \alpha x_{2}$. The reflexive and transitive closure of $\Rightarrow$ is denoted by $\Rightarrow^{*}$. A derivation using the sequence of rules $\pi=r_{1} r_{2} \cdots r_{n}$ is denoted by $\stackrel{\pi}{\Longrightarrow}$ or $\stackrel{r_{1} r_{2} \cdots r_{n}}{\Longrightarrow}$. The language generated by $G$ is defined by $L(G)=\left\{w \in \Sigma^{*} \mid S \Rightarrow^{*} w\right\}$.

A matrix grammar is a quintuple $G=(V, \Sigma, S, M)$ where $V, \Sigma, S$ are defined as for a context-free grammar, $M$ is a finite set of matrices which are finite strings over a set of context-free rules. The language generated by the grammar $G$ consists of all strings $w \in \Sigma^{*}$ such that there is a derivation $S \stackrel{r_{1}}{\stackrel{r_{2} \cdots}{\Longrightarrow} r_{n}} w$ where $r_{1} r_{2} \cdots r_{n}=$ $m_{j_{1}} m_{j_{2}} \cdots m_{j_{k}}$ for some $m_{j_{1}}, m_{j_{2}}, \ldots, m_{j_{k}} \in M$.

A vector grammar is a quintuple $G=(V, \Sigma, S, M)$ whose components are defined as for a matrix grammar. The language generated by the grammar $G$ consists of all strings $w \in \Sigma^{*}$ such that there is a derivation $S \stackrel{r_{1}}{\stackrel{r_{2} \cdots}{\Longrightarrow} r_{n}} w$ where $r_{1} r_{2} \cdots r_{n}=$ $\operatorname{sh}\left(m_{j_{1}}, m_{j_{2}}, \ldots, m_{j_{k}}\right)$ for some $m_{j_{1}}, m_{j_{2}}, \ldots, m_{j_{k}} \in M$.

A semi-matrix grammar is a quintuple $G=(V, \Sigma, S, M)$ whose components are defined as for a matrix grammar. The language generated by the grammar $G$ consists of all strings $w \in \Sigma^{*}$ such that there is a derivation $S \stackrel{r_{1}}{\stackrel{r_{2} \cdots r_{n}}{\Longrightarrow}} w$ where $r_{1} r_{2} \cdots r_{n}=\operatorname{ssh}\left(m_{j_{1}}, m_{j_{2}}, \ldots, m_{j_{k}}\right)$ for some $m_{j_{1}}, m_{j_{2}}, \ldots, m_{j_{k}} \in M$.

A matrix (semi-matrix, vector) grammar $G$ is called without repetitions, if each rule $r$ occurs in $M=\left\{m_{1}, m_{2}, \ldots, m_{n}\right\}$ only once, i.e., $\left|m_{1} m_{2} \cdots m_{n}\right|_{r}=1$.

For each matrix grammar, an equivalent matrix grammar without repetitions can be constructed by adding chain rules.

The families of languages generated by matrix, vector and semi-matrix grammars (with erasing rules) are denoted by MAT, V and $\operatorname{sMAT}_{\mathbf{M A T}}{ }^{\lambda}, \mathbf{V}^{\lambda}$ and sMAT $\left.^{\lambda}\right)$, respectively.

\subsection{Petri nets}

A (place/transition) Petri net $(\mathrm{PN})$ is a construct $N=(P, T, F, \varphi)$ where $P$ and $T$ are disjoint finite sets of places and transitions, respectively, $F \subseteq(P \times T) \cup(T \times P)$ is the set of directed arcs, $\varphi: F \rightarrow\{1,2, \ldots\}$ is a weight function.

A Petri net can be represented by a bipartite directed graph with the node set $P \cup T$ where places are drawn as circles, transitions as boxes and arcs as arrows with labels $\varphi(p, t)$ or $\varphi(t, p)$. If $\varphi(p, t)=1$ or $\varphi(t, p)=1$, the label is omitted.

A mapping $\mu: P \rightarrow\{0,1,2, \ldots\}$ is called a marking. For each place $p \in P$, $\mu(p)$ gives the number of tokens in $p$. Graphically, tokens are drawn as small solid 
dots inside circles. ${ }^{\bullet} x=\{y:(y, x) \in F\}$ and $x^{\bullet}=\{y:(x, y) \in F\}$ are called preand post-sets of $x \in P \cup T$, respectively. The elements of ${ }^{\bullet} t\left({ }^{\bullet} p\right)$ are called input places (transitions) and the elements of $t^{\bullet}\left(p^{\bullet}\right)$ are called output places (transitions) of $t(p)$.

A transition $t \in T$ is enabled by marking $\mu$ iff $\mu(p) \geq \varphi(p, t)$ for all $p \in P$. In this case $t$ can occur (fire). Its occurrence transforms the marking $\mu$ into the marking $\mu^{\prime}$ defined for each place $p \in P$ by $\mu^{\prime}(p)=\mu(p)-\varphi(p, t)+\varphi(t, p)$. A finite sequence $t_{1}, t_{2}, \ldots, t_{k}$ of transitions is called an occurrence sequence enabled at a marking $\mu$ if there are markings $\mu_{1}, \mu_{2}, \ldots, \mu_{k}$ such that $\mu \stackrel{t_{1}}{\longrightarrow} \mu_{1} \stackrel{t_{2}}{\longrightarrow} \ldots \stackrel{t_{k}}{\longrightarrow} \mu_{k}$. In short this sequence can be written as $\mu \stackrel{t_{1} t_{2} \cdots t_{k}}{\longrightarrow} \mu_{k}$ or $\mu \stackrel{\nu}{\rightarrow} \mu_{k}$ where $\nu=t_{1} t_{2} \cdots t_{k}$. For each $1 \leq i \leq k$, the marking $\mu_{i}$ is called reachable from the initial marking $\iota$.

A sequence of places and transitions $\rho=x_{1} x_{2} \cdots x_{n}$ is called a path if and only if no place or transition except $x_{1}$ and $x_{n}$ appears more than once, and $x_{i+1} \in x_{i}^{\bullet}$ for all $1 \leq i \leq n-1 . \operatorname{tr}(\rho)$ denotes the sequence of transitions in a path $\rho$. A path $\rho=x_{1} x_{2} \cdots x_{n}$ is a cycle if $n=1$ or $x_{1}=x_{n}$ and $n \geq 2$. A path $\rho=x_{1} x_{2} \cdots x_{n}$ is a chain if $n=1$ or $x_{1} \neq x_{n}$ and $n \geq 2$. We denote by $P_{\rho}, T_{\rho}, F_{\rho}$ the sets of places, transitions, and arcs of a path $\rho$. Pathes $\rho_{1}$ and $\rho_{2}$ are called disjoint if $P_{\rho_{1}} \cap P_{\rho_{2}}=\emptyset$ and $T_{\rho_{1}} \cap T_{\rho_{2}}=\emptyset$.

A marked Petri net is a system $N=(P, T, F, \varphi, \iota)$ where $(P, T, F, \varphi)$ is a Petri net, $\iota$ is the initial marking. Let $M$ be a set of markings, which will be called final markings. An occurrence sequence $\nu$ of transitions is called successful if it is enabled at the initial marking $\iota$ and finished at a final marking $\tau$ of $M$.

\section{3 cf Petri nets}

The construction of the following type of Petri nets is based on the idea of using similarity between the firing of a transition and the application of a production rule in a derivation in which places are terminal and nonterminal symbols and tokens are separate occurrences of symbols.

Definition 1. Let $G=(V, \Sigma, S, R)$ be a context-free grammar. The cf Petri net with respect to the grammar $G$ is a (labeled marked) Petri net $N=(P, T, F, \varphi, \iota, \beta, \gamma)$ where

- labeling functions $\beta: P \rightarrow(V \cup \Sigma)$ and $\gamma: T \rightarrow R$ are bijections;

- $(p, t) \in F$ iff $\gamma(t)=A \rightarrow w \in R$ where $\beta(p)=A$ and $\varphi(p, t)=1$;

- $(t, p) \in F$ iff $\gamma(t)=A \rightarrow w \in R$ where $\beta(p)=x,|w|_{x}>0$ and $\varphi(t, p)=|w|_{x}$;

- $\iota\left(\beta^{-1}(S)\right)=1$ and $\iota\left(\beta^{-1}(x)\right)=0$ for all $x \in(V \cup \Sigma) \backslash\{S\}$.

Example 1. Let $G_{1}$ be a context-free grammar with the rules: $S \rightarrow A B, A \rightarrow$ $a A|b A| a|b, B \rightarrow a B| b B|a| b$. (the other components of the grammar can be seen 


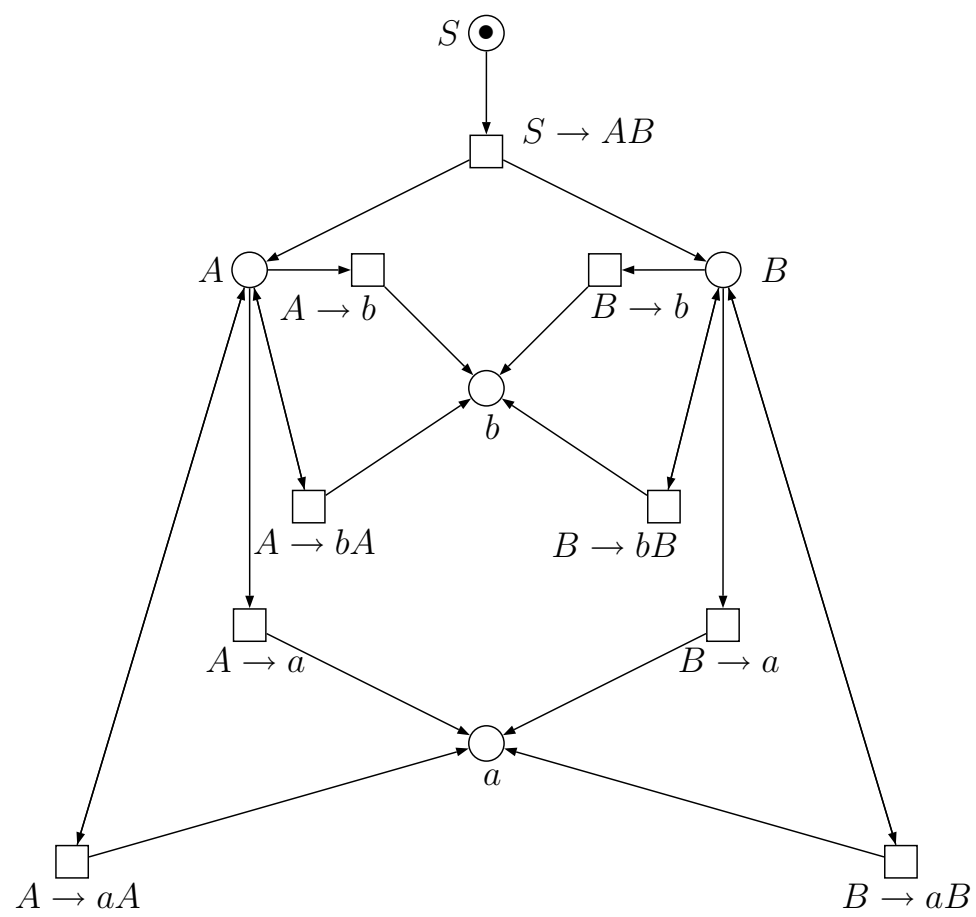

Fig. 1. A cf Petri net $N$.

from these rules). Figure 1 illustrates the corresponding of Petri net $N$. Obviously, $L\left(G_{1}\right)=\{a, b\}^{+}$.

The following proposition, which directly follows from the definition, shows the similarity between terminal derivations in a context-free grammar and successful occurrences in the corresponding cf Petri net.

Proposition 1. Let $N=(P, T, F, \varphi, \iota, \beta, \gamma)$ be the cf Petri net with respect to a

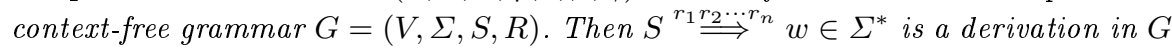
iff $\iota \stackrel{t_{1} t_{2} \cdots t_{n}}{\longrightarrow} \tau$ is an occurrence sequence of transitions in $N$ such that $\gamma\left(t_{1} t_{2} \cdots t_{n}\right)=$ $r_{1} r_{2} \cdots r_{n}$ and $\tau\left(\beta^{-1}(x)\right)=0$ for all $x \in V$.

\section{Petri Net Controlled Grammars}

In this section we construct Petri net control mechanisms and define Petri net controlled grammars. 


\subsection{Chain, cyclic and supervised cyclic controls}

We add new places and arcs, called control places and arcs, to a cf Petri net in such a way that the new places with input and output transitions make up chains or cycles. Let $N=(P, T, F, \varphi, \iota, \beta, \gamma)$ be a cf Petri net with respect to a context-free grammar $G=(V, \Sigma, S, R)$. Let $P_{V}=\left\{p \mid p=\beta^{-1}(x), x \in V\right\}$ and $P_{\Sigma}=\{p \mid p=$ $\left.\beta^{-1}(x), x \in \Sigma\right\}$. We use these notations in all definitions hereinafter.

Chain control. Let $\Pi=\left\{\rho_{1}, \rho_{2}, \ldots, \rho_{n}\right\}$ be a set of disjoint chains where for all $\rho \in \Pi, \rho=t_{1} p_{1} t_{2} p_{2} \cdots p_{k-1} t_{k}$ where $T_{\rho}=\left\{t_{1}, t_{2}, \ldots, t_{k}\right\} \subseteq T$ and $P_{\rho}=$ $\left\{p_{1}, p_{2}, \ldots, p_{k-1}\right\}, F_{\rho}=\left\{\left(t_{i}, p_{i}\right) \mid 1 \leq i \leq k-1\right\} \cup\left\{\left(p_{i}, t_{i+1}\right) \mid 1 \leq i \leq k-1\right\}$ are the set of new places and arcs, respectively. Let

$$
P_{\Pi}=\bigcup_{\rho \in \Pi} P_{\rho}, \quad T_{\Pi}=\bigcup_{\rho \in \Pi} T_{\rho}, \quad F_{\Pi}=\bigcup_{\rho \in \Pi} F_{\rho} .
$$

Without loss of generality we assume that $T=T_{\Pi}$ ( since each transition of $T$ can be considered as a chain or a cycle).

Definition 2. A chain-cf Petri net is a system $\hat{N}=(\hat{P}, T, \hat{F}, \hat{\varphi}, \hat{\iota}, \hat{\beta}, \gamma, M)$ where

- $\hat{P}=P \cup P_{\Pi} \quad$ and $\quad \hat{F}=F \cup F_{\Pi}$,

- the weight function $\hat{\varphi}$ is defined by

$$
\hat{\varphi}(x, y)= \begin{cases}\varphi(x, y) & \text { if }(x, y) \in F \\ 1 & \text { if }(x, y) \in F_{\Pi},\end{cases}
$$

- the initial marking $\hat{\imath}$ is defined by

$$
\hat{\iota}(p)= \begin{cases}\iota(p) & \text { if } p \in P \\ 0 & \text { if } p \in P_{\Pi},\end{cases}
$$

- the labeling function $\hat{\beta}: \hat{P} \rightarrow V \cup \Sigma \cup\{\lambda\}$ is defined by

$$
\hat{\beta}(p)= \begin{cases}\beta(p) & \text { if } p \in P \\ \lambda & \text { if } p \in P_{\Pi},\end{cases}
$$

- $M$ is a set of final markings where for each $\tau \in M, \tau(p)=0$ for all $p \in \hat{P}-P_{\Sigma}$.

Example 2. Figure 2 illustrates a chain-cf Petri net $\hat{N}$ which is based on the contextfree grammar introduced in Example 1.

According to the construction of a chain-cf Petri net $\hat{N}$, the firing of its transitions follows the rules below: 


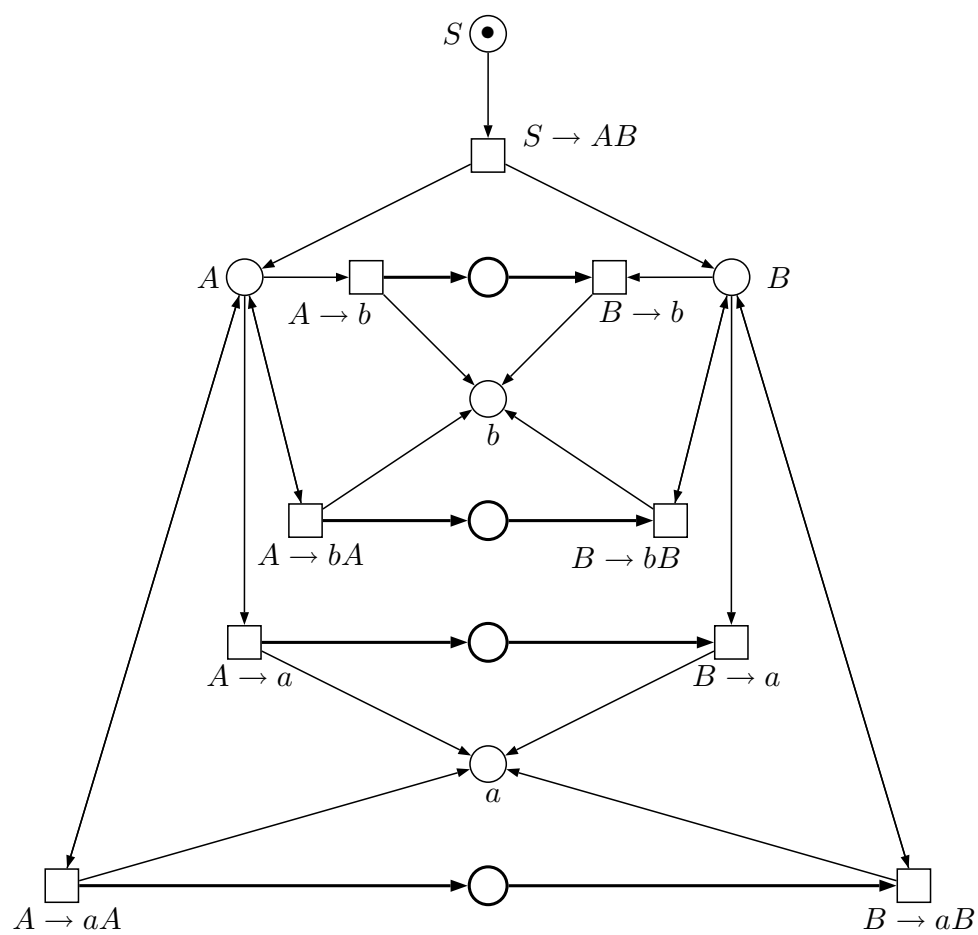

Fig. 2. A chain-cf Petri net $\hat{N}$.

- for each chain $\rho=t_{1} p_{1} t_{2} p_{2} \cdots t_{n-1} p_{n-1} t_{n} \in \Pi$, the firing of its transitions starts with the firing of transition $t_{1}$, and $t_{1}$ can occur any time (when it is enabled), it does not depend on the next transitions in the sequence $\operatorname{tr}(\rho)$;

- transition $t_{i}$ can occur if $t_{i-1}, 1<i \leq n$, has occurred, and for each occurrence of sequence $\sigma$ of transitions of $\hat{N},|\sigma|_{t_{i-1}} \geq|\sigma|_{t_{i}}$. Moreover, if $\sigma$ is successful then $|\sigma|_{t_{1}}=|\sigma|_{t_{2}}=\cdots=|\sigma|_{t_{n}}$;

- the transitions of disjoint chains can occur concurrently since the places of a chain merely control the firing of the transitions of the chain.

Consequently,

Proposition 2. For each successful occurrence sequence transitions $\sigma=t_{1} t_{2} \cdots t_{n}$ of a chain-cf Petri net $\hat{N}, \sigma=\operatorname{sh}\left(\operatorname{tr}\left(\rho_{i_{1}}\right), \operatorname{tr}\left(\rho_{i_{2}}\right), \ldots, \operatorname{tr}\left(\rho_{i_{s}}\right)\right)$ for some (not necessarily different) chains $\rho_{i_{1}}, \rho_{i_{2}}, \ldots, \rho_{i_{s}} \in \Pi, s \geq 1$. 
Cyclic control. Let $\Pi=\left\{\rho_{1}, \rho_{2}, \ldots, \rho_{n}\right\}$ be a set of disjoint cycles where for all $\rho \in$ $\Pi, \rho=p_{1} t_{1} p_{2} t_{2} \cdots p_{k} t_{k} p_{1}$ where $T_{\rho}=\left\{t_{1}, t_{2}, \ldots, t_{k}\right\} \subseteq T$ and $P_{\rho}=\left\{p_{1}, p_{2}, \ldots, p_{k}\right\}$, $F_{\rho}=\left\{\left(p_{i}, t_{i}\right) \mid 1 \leq i \leq k\right\} \cup\left\{\left(t_{i}, p_{i+1}\right) \mid 1 \leq i \leq k-1\right\} \cup\left\{\left(t_{k}, p_{1}\right)\right\}$ are the set of new places and arcs, respectively. Let

$$
P_{\Pi}=\bigcup_{\rho \in \Pi} P_{\rho}, \quad T_{\Pi}=\bigcup_{\rho \in \Pi} T_{\rho}, \quad F_{\Pi}=\bigcup_{\rho \in \Pi} F_{\rho} .
$$

Definition 3. A cyclic-cf Petri net is a system $\check{N}=(\check{P}, T, \check{F}, \check{\varphi}, \check{\iota}, \check{\beta}, \gamma, M)$ where

- $\hat{P}=P \cup P_{\Pi} \quad$ and $\quad \hat{F}=F \cup F_{\Pi}$,

- the weight function $\check{\varphi}$ is defined by

$$
\check{\varphi}(x, y)= \begin{cases}\varphi(x, y) & \text { if }(x, y) \in F \\ 1 & \text { if }(x, y) \in F_{\Pi},\end{cases}
$$

- the initial marking $\hat{\imath}$ is defined by

$$
\check{\iota}(p)= \begin{cases}\iota(p) & \text { if } p \in P \\ 1 & \text { if } p=p_{1} \in P_{\rho}, \rho \in \Pi \\ 0 & \text { if } p \in P_{\rho}-\left\{p_{1}\right\}, \rho \in \Pi,\end{cases}
$$

- the labeling function $\check{\beta}: \check{P} \rightarrow V \cup \Sigma \cup\{\lambda\}$ is defined by

$$
\check{\beta}(p)= \begin{cases}\beta(p) & \text { if } p \in P \\ \lambda & \text { if } p \in P_{\Pi},\end{cases}
$$

- $M$ is a set of final markings where for each $\tau \in M$,

$$
\check{\tau}(p)= \begin{cases}0 & \text { if } p \in P_{V} \\ 1 & \text { if } p=p_{1} \in P_{\rho}, \rho \in \Pi \\ 0 & \text { if } p \in P_{\rho}-\left\{p_{1}\right\}, \rho \in \Pi .\end{cases}
$$

Example 3. Figure 3 illustrates a cyclic-cf Petri net $\check{N}$ which is based on the contextfree grammar given in Example 1.

According to the construction of a cyclic-cf Petri net $\check{N}$, the firing of its transitions follows the rules below:

- for each cycle $\rho=p_{1} t_{1} p_{2} t_{2} \cdots p_{n} t_{n} p_{1} \in \Pi$, the firing of its transitions starts with the firing of transition $t_{1}$, and $t_{1}$ can occur next when transitions $t_{2}, t_{3}, \ldots, t_{n}$ have occurred in the defined order;

- for each occurrence of sequence $\sigma$ of transitions of $\check{N},|\sigma|_{t_{i-1}}=|\sigma|_{t_{i}}$ or $|\sigma|_{t_{i-1}}=$ $|\sigma|_{t_{i}}+1$, and if $\sigma$ is successful then $|\sigma|_{t_{1}}=|\sigma|_{t_{2}}=\cdots=|\sigma|_{t_{n}}$ 


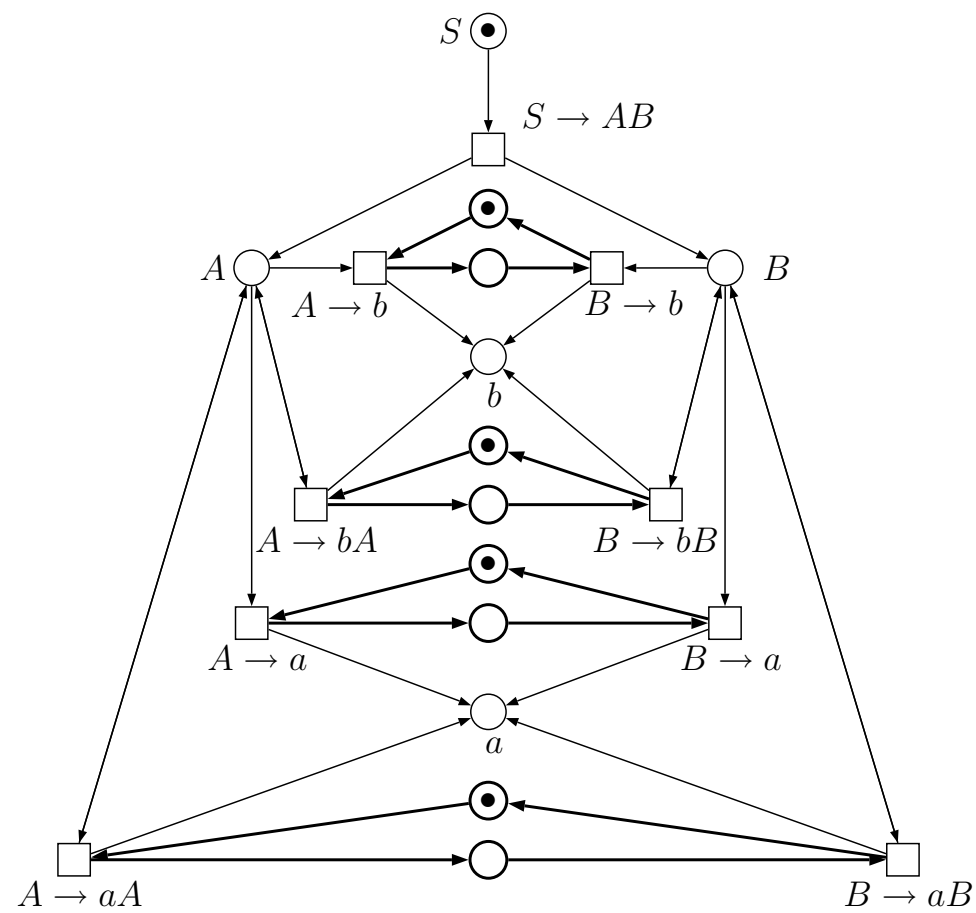

Fig. 3. A cyclic-cf Petri net $\check{N}$.

- the transitions of disjoint cycles can occur concurrently since the places of a cycle merely control the firing of the transitions of the cycle while the occurrence of the transitions of the same cycle a second time can start when the first occurrence of the transitions finishes.

Consequently,

Proposition 3. For each successful occurrence sequence transitions $\sigma=t_{1} t_{2} \cdots t_{n}$ of a cyclic-cf Petri net $\check{N}, \sigma=\operatorname{ssh}\left(\operatorname{tr}\left(\rho_{i_{1}}\right), \operatorname{tr}\left(\rho_{i_{2}}\right), \ldots, \operatorname{tr}\left(\rho_{i_{s}}\right)\right)$ for some (not necessarily different) chains $\rho_{i_{1}}, \rho_{i_{2}}, \ldots, \rho_{i_{s}} \in \Pi, s \geq 1$.

Supervised cyclic control. Let $\Pi=\left\{\rho_{1}, \rho_{2}, \ldots, \rho_{n}\right\}$ be a set of cycles such that $P_{\rho_{1}} \cap P_{\rho_{2}} \cap \cdots \cap P_{\rho_{n}}=\left\{p_{0}\right\}$ where for all $\rho \in \Pi, \rho=p_{0} t_{1} p_{1} t_{2} \cdots p_{k-1} t_{k} p_{0}$ where $T_{\rho}=\left\{t_{1}, t_{2}, \ldots, t_{k}\right\} \subseteq T$ and $P_{\rho}=\left\{p_{0}, p_{1}, \ldots, p_{k-1}\right\}, F_{\rho}=\left\{\left(p_{i}, t_{i+1}\right) \mid 0 \leq i \leq\right.$ $k-1\} \cup\left\{\left(t_{i}, p_{i}\right) \mid 1 \leq i \leq k-1\right\} \cup\left\{\left(t_{k}, p_{0}\right)\right\}$ are the set of new places and arcs, respectively. Let 


$$
P_{\Pi}=\bigcup_{\rho \in \Pi} P_{\rho}, \quad T_{\Pi}=\bigcup_{\rho \in \Pi} T_{\rho}, \quad F_{\Pi}=\bigcup_{\rho \in \Pi} F_{\rho} .
$$

Definition 4. A system $\tilde{N}=(\tilde{P}, T, \tilde{F}, \tilde{\varphi}, \tilde{\iota}, \tilde{\beta}, \gamma, M)$ is called a supervised-cyclic-cf Petri net where

- $\quad \tilde{P}=P \cup P_{\Pi} \quad$ and $\quad \tilde{F}=F \cup F_{\Pi}$,

- the weight function $\check{\varphi}$ is defined by

$$
\tilde{\varphi}(x, y)= \begin{cases}\varphi(x, y) & \text { if }(x, y) \in F \\ 1 & \text { if }(x, y) \in F_{\Pi},\end{cases}
$$

- the initial marking $\tilde{\iota}$ is defined by

$$
\tilde{\iota}(p)= \begin{cases}\iota(p) & \text { if } p \in P \\ 1 & \text { if } p=p_{0} \\ 0 & \text { if } p \in P_{\rho}-\left\{p_{0}\right\}, \rho \in \Pi,\end{cases}
$$

- the labeling function $\tilde{\beta}: \tilde{P} \rightarrow V \cup \Sigma \cup\{\lambda\}$ is defined by

$$
\tilde{\beta}(p)= \begin{cases}\beta(p) & \text { if } p \in P \\ \lambda & \text { if } p \in P_{\Pi},\end{cases}
$$

- $M$ is a set of final markings where for each $\tau \in M$,

$$
\tilde{\tau}(p)= \begin{cases}0 & \text { if } p \in P_{V} \\ 1 & \text { if } p=p_{0} \\ 0 & \text { if } p \in P_{\rho}-\left\{p_{0}\right\}, \rho \in \Pi .\end{cases}
$$

Example 4. Figure 4 illustrates a supervised-cyclic-cf Petri net $\tilde{N}$ which is based on the context-free grammar given in Example 1.

According to the construction of a supervised-cyclic-cf Petri net $\tilde{N}$, the firing of its transitions follows the rules below:

- for each cycle $\rho=p_{0} t_{1} p_{1} t_{2} \cdots p_{n_{1}} t_{n} p_{0} \in \Pi$, the firing of its transitions starts with the firing of transition $t_{1}$, and $t_{1}$ can occur next when transitions $t_{2}, t_{3}, \ldots, t_{n}$ have occurred in the defined order;

- for each successful occurrence of sequence $\sigma$ of transitions of $\tilde{N},|\sigma|_{t_{1}}=|\sigma|_{t_{2}}=$ $\cdots=|\sigma|_{t_{n}}$;

- if the occurrence of the transitions of a cycle $\rho_{1}$ has started then the occurrence of the transitions of a second (not necessarily different) cycle $\rho_{2}$ can start when all transitions of $\rho_{1}$ have fired.

Consequently,

Proposition 4. For each successful occurrence sequence transitions $\sigma=t_{1} t_{2} \cdots t_{n}$ of a supervised-cyclic-cf Petri net $\tilde{N}, \sigma=\operatorname{tr}\left(\rho_{i_{1}}\right) \operatorname{tr}\left(\rho_{i_{2}}\right) \cdots \operatorname{tr}\left(\rho_{i_{s}}\right)$ for some (not necessarily different) cycles $\rho_{i_{1}}, \rho_{i_{2}}, \ldots, \rho_{i_{s}} \in \Pi, s \geq 1$. 


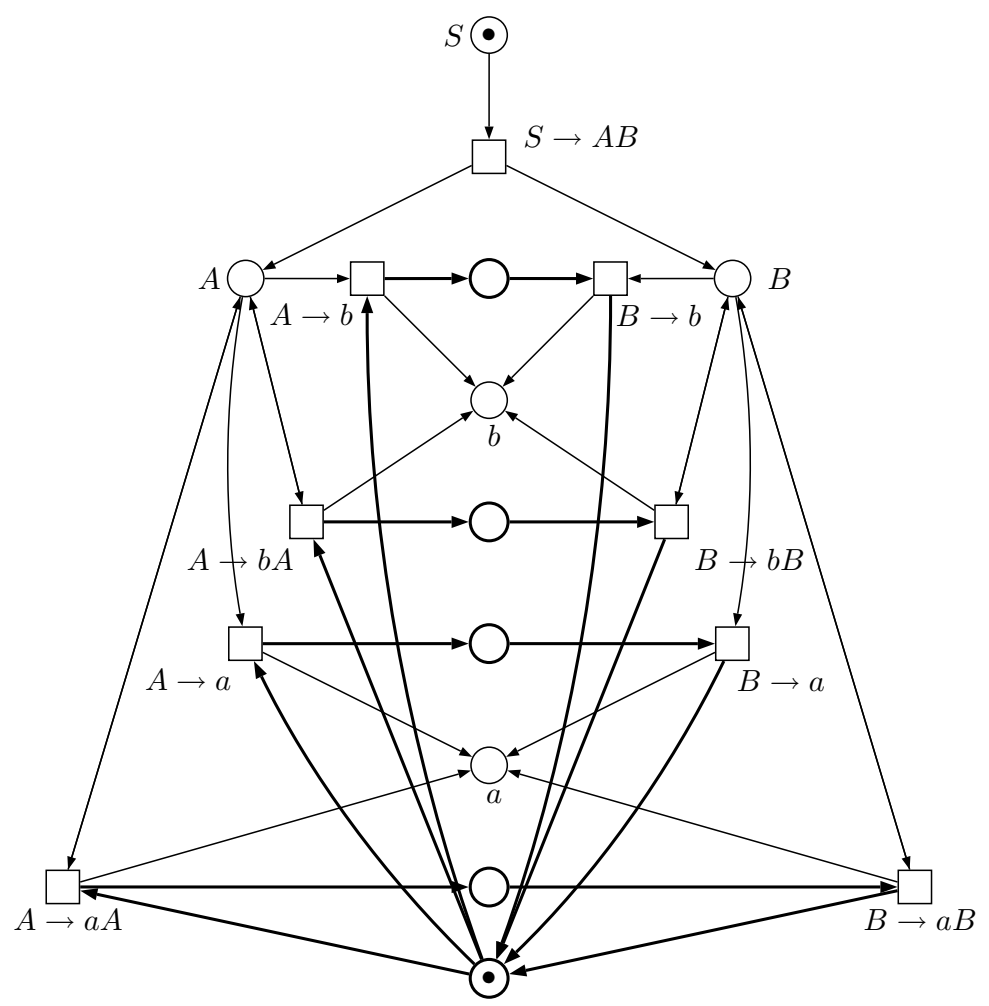

Fig. 4. A supervised-cyclic-cf Petri net $\tilde{N}$.

\subsection{Grammars and examples}

In this section we define the grammars controlled by the Petri nets introduced in the section above.

Definition 5. A chain- (respectively, cyclic-, supervised-cyclic-) cf Petri net controlled grammar (in short a ch- (c-, sc-) $P N$ controlled grammar) is a tuple $G=(V, \Sigma, S, R, \hat{N})(G=(V, \Sigma, S, R, \check{N}), G=(V, \Sigma, S, R, \tilde{N}))$ where $V, \Sigma, S$, $R$ are defined as for a context-free grammar and $\hat{N}(\check{N}, \tilde{N})$ is a chain- (cyclic-, supervised-cyclic-) cf Petri net with respect to the context-free grammar $(V, \Sigma, S, R)$.

Definition 6. The language generated by a ch- (c-, sc-) Petri net controlled grammar $G$, denoted by $L(G)$, consists of all strings $w \in \Sigma^{*}$ such that there is a derivation $S \stackrel{r_{1}}{\stackrel{r_{2} \cdots r_{k}}{\Longrightarrow}} w \in \Sigma^{*}$ and a successful occurrence sequence of transitions $\nu=t_{1} t_{2} \cdots t_{k}$ 
of $\hat{N}(\check{N}, \tilde{N})$ such that $r_{1} r_{2} \cdots r_{k}=\hat{\gamma}\left(t_{1} t_{2} \cdots t_{k}\right) \quad\left(r_{1} r_{2} \cdots r_{k}=\check{\gamma}\left(t_{1} t_{2} \cdots t_{k}\right)\right.$, $r_{1} r_{2} \cdots r_{k}=\tilde{\gamma}\left(t_{1} t_{2} \cdots t_{k}\right)$, respectively).

Example 5. Let $G_{2}=(V, \Sigma, S, R, \hat{N})$ be a ch-Petri net controlled grammar where components $V, \Sigma, S, R$ are defined as for the context-free grammar $G_{2}$ in Example 1 , and $\hat{N}$ is the chain-cf Petri net in Figure 2.

After transition $t_{0}=\hat{\beta}^{-1}(S \rightarrow A B)$ fires, transitions $t_{1}=\hat{\beta}^{-1}(A \rightarrow a A)$, $t_{3}=\hat{\beta}^{-1}(A \rightarrow b A), t_{2}=\hat{\beta}^{-1}(A \rightarrow a)$, and $t_{4}=\hat{\beta}^{-1}(A \rightarrow b)$ are enabled. Transitions $t_{1}$ and (or) $t_{3}$ can occur several times and in any order, and the corresponding control places receive as many tokens as the numbers of occurrences of these transitions. Then transitions $t_{5}=\hat{\beta}^{-1}(B \rightarrow a B)$ and $t_{7}=\hat{\beta}^{-1}(B \rightarrow b B)$ occur as many times as $t_{1}$ and (or) $t_{3}$ occur. To go a final marking, transition $t_{2}=\hat{\beta}^{-1}(A \rightarrow a)$ or $t_{4}=\hat{\beta}^{-1}(A \rightarrow b)$ occurs, then, respectively, transition $t_{6}=\hat{\beta}^{-1}(B \rightarrow a)$ or $t_{8}=\hat{\beta}^{-1}(B \rightarrow b)$ occurs. One can see that $G_{2}$ generates a vector language

$$
L\left(G_{2}\right)=\left\{w x w^{\prime} x \mid x \in\{a, b\}, w \in\{a, b\}^{*}, w^{\prime} \in \operatorname{Perm}(w)\right\} .
$$

Example 6. We consider the same context-free grammar as that in Example 1 with the cyclic-cf Petri net $\check{N}$ in Figure 3 (the transitions of $\check{N}$ have the same labels as those of $\hat{N})$, i.e., $G_{3}=(V, \Sigma, S, R, \check{N})$.

After transition $t_{0}$ occurs transitions $t_{1}, t_{2}, t_{3}, t_{4}$ are enabled. The following cases may arise: (1) the sequence $t_{1} t_{5}$ or (and) $t_{3} t_{7}$ occurs many times (in any order); (2) the sequence $t_{1} t_{3}$ or $t_{3} t_{1}$ occurs, then $t_{5} t_{7}$ or $t_{7} t_{5}$ occurs; (3) cases (1) and (2) repeat in any order; (4) to go to a final marking the sequence $t_{2} t_{6}$ or $t_{4} t_{8}$ occurs. It is easy to see that $G_{4}$ generates a semi-matrix language

$$
\begin{gathered}
L\left(G_{3}\right)=\left\{w_{1}\{\lambda, a b, b a\} w_{2}\{\lambda, a b, b a\} \cdots x \cdot w_{1}\{\lambda, a b, b a\} w_{2}\{\lambda, a b, b a\} \cdots x \mid\right. \\
\left.x \in\{a, b\}, w_{1}, w_{2}, \ldots \in\{a, b\}^{*}\right\} .
\end{gathered}
$$

Example 7. Let $G_{4}$ consist of context-free components of the grammar $G_{1}$ (Example 1) and the supervised-cyclic-cf Petri net $\tilde{N}$ in Figure 4 (it can be seen that the transitions preserve the labels).

The execution of $\tilde{N}$ starts with the occurrence of $t_{0}$, and transitions $t_{1}, t_{2}, t_{3}$, and $t_{4}$ are enabled. Next, only one of them can occur, enabling, respectively, $t_{5}, t_{6}, t_{7}$, and $t_{8}$. The sequence $t_{1} t_{5}$ or $t_{3} t_{7}$ occurs many times (in any order). To go to a final marking one of the sequences $t_{2} t_{6}, t_{4} t_{8}$ occurs. We can see that $G_{3}$ generates a matrix language

$$
L\left(G_{3}\right)=\left\{w w \mid w \in\{a, b\}^{+}\right\} .
$$

We denote the families of languages generated by ch- $\{c-$, sc- $\}$ Petri net controlled grammars (with erasing rules) by CHPN, CPN, SCPN $\left(\mathbf{C H P N}{ }^{\lambda}, \mathbf{C P N}^{\lambda}\right.$, $\operatorname{SCPN}^{\lambda}$ ), respectively. 


\section{Generative Capacity}

In this section we show that the introduced Petri net controlled grammars simulate some well-known regulated grammars.

\section{Lemma 1.}
(1) $\mathbf{V} \subseteq \mathbf{C H P N}$ and $\mathbf{V}^{\lambda} \subseteq \mathbf{C H P N} \mathbf{N}^{\lambda}$,
(2) $\mathbf{s M A T} \subseteq \mathbf{C P N}$ and $\mathbf{s M A T}^{\lambda} \subseteq \mathbf{C P N}^{\lambda}$,
(3) $\mathbf{M A T} \subseteq \mathbf{S C P N}$ and $\mathbf{M A T}^{\lambda} \subseteq \mathbf{S C P N}^{\lambda}$.

Proof. Let $G=(V, \Sigma, S, M)$ be a vector (semi-matrix, matrix) grammar where $M=\left\{m_{1}, m_{2}, \ldots, m_{k}\right\}$. Without loss of generality we can assume that $G$ is a grammar without repetition. Let $R$ be the set of all rules of $M$. We construct a cf Petri net $N=(P, T, F, \varphi, \iota, \beta, \gamma)$ (with the notions of Def. 1 ).

(CHAIN): For each matrix $m=r_{1} r_{2} \cdots r_{n} \in M$ we define a chain

$\rho_{m}=t_{1} p_{1} t_{2} p_{2} \cdots t_{n-1} p_{n-1} t_{n}$ where $p_{1}, p_{2}, \ldots, p_{n-1}$ are new places and $\gamma\left(t_{i}\right)=$ $r_{i}, 1 \leq i \leq n$. Then $\gamma(\operatorname{tr}(\rho))=m$ since $\gamma$ is a bijection. Let $\Pi=\left\{\rho_{m_{1}}, \rho_{m_{2}}, \ldots\right.$, $\left.\rho_{m_{k}}\right\}$. As chains of $\Pi$ are disjoint, we construct a chain-cf Petri net $\hat{N}$ (with notions of Def. 2), and define ch-Petri net controlled grammar $\hat{G}=(V, \Sigma, S, R, \hat{N})$.

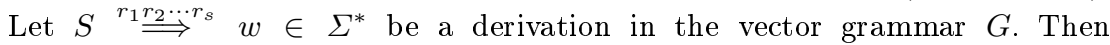
$r_{1} r_{2} \cdots r_{s}=\operatorname{sh}\left(m_{1}^{\prime}, m_{2}^{\prime}, \ldots, m_{l}^{\prime}\right)$ for some $m_{1}^{\prime}, m_{2}^{\prime}, \ldots, m_{l}^{\prime} \in M$. By bijection $\gamma$

$$
\begin{aligned}
t_{1} t_{2} \cdots t_{s} & =\gamma^{-1}\left(r_{1} r_{2} \cdots r_{s}\right) \\
& =\gamma^{-1}\left(\operatorname{sh}\left(m_{1}^{\prime}, m_{2}^{\prime}, \ldots, m_{l}^{\prime}\right)\right) \\
& =\operatorname{sh}\left(\gamma^{-1}\left(m_{1}^{\prime}\right), \gamma^{-1}\left(m_{2}^{\prime}\right), \ldots, \gamma^{-1}\left(m_{l}^{\prime}\right)\right) \\
& =\operatorname{sh}\left(\operatorname{tr}\left(\rho_{1}^{\prime}\right), \operatorname{tr}\left(\rho_{2}^{\prime}\right), \ldots, \operatorname{tr}\left(\rho_{l}^{\prime}\right)\right) .
\end{aligned}
$$

Hence, $t_{1} t_{2} \cdots t_{s}$ is a successful occurrence sequence in $\hat{N}$, and $S \stackrel{r_{1}}{\stackrel{r_{2}}{\Longrightarrow} r_{s}} w \in \Sigma^{*}$ is also derivation in $\hat{G}$, i.e., $L(G) \subseteq L(\hat{G})$. The inverse inclusion can also be shown by backtracking (1).

(CYCLE): For each matrix $m=r_{1} r_{2} \cdots r_{n} \in M$ we define a cycle

$\rho_{m}=p_{1} t_{1} p_{2} \cdots p_{n} t_{n} p_{1}$ where $p_{1}, p_{2}, \ldots, p_{n}$ are new places and $\gamma\left(t_{i}\right)=r_{i}, 1 \leq$ $i \leq n$. Since $\gamma$ is a bijection, $\gamma(\operatorname{tr}(\rho))=m$. Let $\Pi=\left\{\rho_{m_{1}}, \rho_{m_{2}}, \ldots, \rho_{m_{k}}\right\}$. As $\Pi$ consists of disjoint cycles, we construct a cyclic-cf Petri net $N$ (with notions of Def. 3) and define c-Petri net controlled grammar $\check{G}=(V, \Sigma, S, R, \check{N})$.

Let $S \stackrel{r_{1}}{\stackrel{r_{2} \cdots r_{s}}{\Longrightarrow}} w \in \Sigma^{*}$ be a derivation in the semi-matrix grammar $G$. Then $r_{1} r_{2} \cdots r_{s}=\operatorname{ssh}\left(m_{1}^{\prime}, m_{2}^{\prime}, \ldots, m_{l}^{\prime}\right)$ for some $m_{1}^{\prime}, m_{2}^{\prime}, \ldots, m_{l}^{\prime} \in M$. By bijection $\gamma$, 


$$
\begin{aligned}
t_{1} t_{2} \cdots t_{s} & =\gamma^{-1}\left(r_{1} r_{2} \cdots r_{s}\right) \\
& =\gamma^{-1}\left(\operatorname{ssh}\left(m_{1}^{\prime}, m_{2}^{\prime}, \ldots, m_{l}^{\prime}\right)\right) \\
& =\operatorname{ssh}\left(\gamma^{-1}\left(m_{1}^{\prime}\right), \gamma^{-1}\left(m_{2}^{\prime}\right), \ldots, \gamma^{-1}\left(m_{l}^{\prime}\right)\right) \\
& =\operatorname{ssh}\left(\operatorname{tr}\left(\rho_{1}^{\prime}\right), \operatorname{tr}\left(\rho_{2}^{\prime}\right), \ldots, \operatorname{tr}\left(\rho_{l}^{\prime}\right)\right) .
\end{aligned}
$$

It follows that $t_{1} t_{2} \cdots t_{s}$ is a successful occurrence sequence in $\check{N}$. Therefore, $L(G) \subseteq L(\check{G})$. The inverse inclusion can be shown in similar way.

(SUPERVISED CYCLE): For each matrix $m=r_{1} r_{2} \cdots r_{n} \in M$ we define a cycle $\rho_{m}=p_{0} t_{1} p_{1} \cdots p_{n-1} t_{n} p_{0}$ where $p_{0}, p_{1}, \ldots, p_{n-1}$ are new places and $\gamma\left(t_{i}\right)=r_{i}$, $1 \leq i \leq n$. Since $\gamma$ is a bijection, $\gamma(\operatorname{tr}(\rho))=m$. Let $\Pi=\left\{\rho_{m_{1}}, \rho_{m_{2}}, \ldots, \rho_{m_{k}}\right\}$. As the cycles of $\Pi$ have the unique common place $p_{0}$, we construct a supervisedcyclic-cf Petri net $\tilde{N}$ (with notions of Def. 4) and define sc-Petri net controlled grammar $\tilde{G}=(V, \Sigma, S, R, \tilde{N})$.

Let $S \stackrel{r_{1}}{\stackrel{r_{2} \cdots r_{s}}{\Longrightarrow}} w \in \Sigma^{*}$ be a derivation in the matrix grammar $G$. Then $r_{1} r_{2} \cdots r_{s}=m_{1}^{\prime} m_{2}^{\prime} \cdots m_{l}^{\prime}$ ) for some $m_{1}^{\prime}, m_{2}^{\prime}, \ldots, m_{l}^{\prime} \in M$. By bijection $\gamma$,

$$
\begin{aligned}
t_{1} t_{2} \cdots t_{s} & =\gamma^{-1}\left(r_{1} r_{2} \cdots r_{s}\right) \\
& =\gamma^{-1}\left(m_{1}^{\prime} m_{2}^{\prime} \cdots m_{l}^{\prime}\right) \\
& =\gamma^{-1}\left(m_{1}^{\prime}\right) \gamma^{-1}\left(m_{2}^{\prime}\right) \cdots \gamma^{-1}\left(m_{l}^{\prime}\right) \\
& =\operatorname{tr}\left(\rho_{1}^{\prime}\right) \operatorname{tr}\left(\rho_{2}^{\prime}\right) \cdots \operatorname{tr}\left(\rho_{l}^{\prime}\right) .
\end{aligned}
$$

Thus $t_{1} t_{2} \cdots t_{s}$ is a successful occurrence sequence in $\check{N}$ and $L(G) \subseteq L(\check{G})$. The inverse inclusion can be shown using similar arguments.

By analogous considerations, it can be shown that:

\section{Lemma 2.}

(1) $\mathbf{C H P N} \subseteq \mathbf{V}$ and $\mathbf{C H P N}^{\lambda} \subseteq \mathbf{V}^{\lambda}$,

(2) $\mathbf{C P N} \subseteq \mathbf{s M A T}$ and $\mathbf{C P N}^{\lambda} \subseteq \mathbf{s M A T}^{\lambda}$,

(3) $\mathbf{S C P N} \subseteq$ MAT and $\mathbf{S C P N}^{\lambda} \subseteq \mathbf{M A T}^{\lambda}$.

The results of Lemmas 1, 2, Theorem 2.1.2 ([4]) and Theorem 7 ([14]) can be summarized in the following theorem.

Theorem 1. The inclusions presented in Figure 5 hold. 


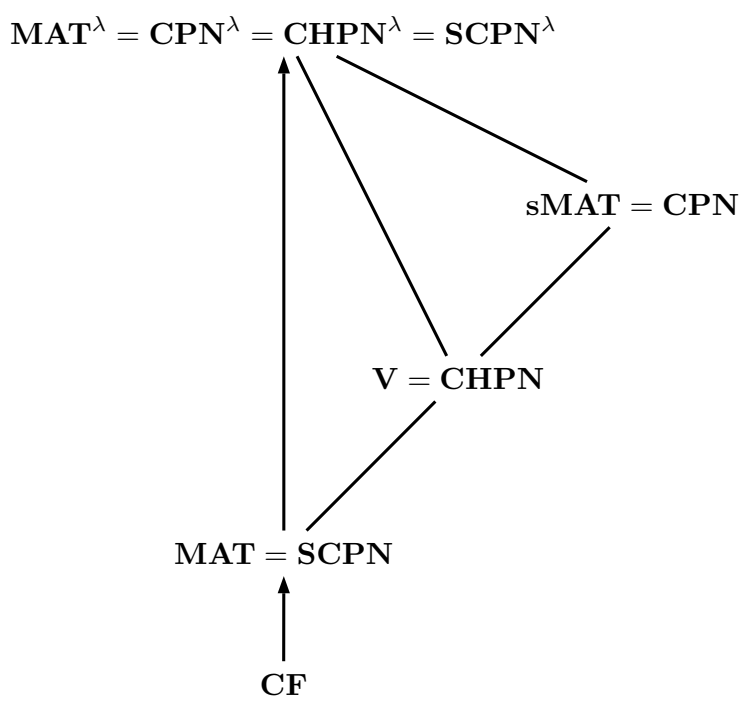

Fig. 5. If two families are connected by a line (an arrow), then the upper family includes (includes properly) the lower family.

\section{References}

1. Abraham, A. (1965). Some questions of phrase-structure grammars. Computational Linguistics, 4: 61-70.

2. Ceska, M. and V. Marek (2001). Petri nets and random-context grammars. In Proceedings of the 35th Spring Conference: Modeling and Simulation of Systems (MOSIS'01), pp. 145-152. MARQ Ostrava Hardec and Moravici.

3. Cremers, A.B. and O. Mayer (1973). On matrix languages. Information Control, 23: $86-96$.

4. Dassow, J. and Gh. Păun (1989). Regulated rewriting in formal language theory. Berlin: Springer.

5. Ginsburg, S. and E.H. Spanier (1968). Control sets on grammars. Math. Syst. Th., 2: 159-177.

6. Hack, M. (1976). Petri net languages. MIT Laboratory of Computer Science Technical report 159. Cambridge

7. Hopcroft, J.E. and J.D. Ullman (1990). Introduction to automata theory, languages, and computation. Reading: Addison-Wesley.

8. Păun, Gh. (1980). A new generative device: valence grammars. Rev. Roum. Math. Pures Appl., 25: 911-924.

9. Reisig, W. and G. Rozenberg (eds.) (1998). Lectures on Petri nets I: Basic models. Lecture Notes in Computer science, 1491. Berlin: Springer. 
10. Rosenkratz, D.J. (1969). Programmed grammars and classes of formal languages. Journal of the ACM, 16: 107-131.

11. Rozenberg, G. and A. Salomaa (1997). Handbook of formal languages. Berlin: Springer.

12. Starke, P.H. (1978). Free Petri net languages. Lecture Notes in Computer Science, 64: 506-515.

13. Starke, P.H. (1980). Petri-Netze. Deutscher Verlag der Wissenschaften.

14. Turaev, S. (2006). Semi-matrix grammars. In Proceedings 2nd Doctoral Workshop on Mathematical and Engineering Methods in Computer Science MEMICS2006, pp. 245-252.

15. Van Der Walt, A.P.J. (1970). Random context grammars. In Proceedings of the Symposium on Formal Languages. Oberwolfach. 